\title{
Anomalous scaling of anisotropy of second-order moments in a model of a randomly advected solenoidal vector field
}

\author{
Kyo Yoshida and Yukio Kaneda \\ Department of Computational Science and Engineering, Graduate School of Engineering, Nagoya University, Chikusa-ku, \\ Nagoya 464-8603, Japan
}

(Received 25 May 2000; published 22 December 2000)

\begin{abstract}
A model of randomly advected solenoidal field is presented. The model is formally derived by a linearization of the Navier-Stokes equation with respect to the perturbation to a basic state and by assuming the characteristic time scale of the basic state to be very short. The model includes a nonlocal (in space) effect through a pressurelike term that keeps the advected field solenoidal, but still yields exact equations for multipoint moments. The advecting field is assumed to be statistically homogeneous and isotropic with zero mean and structure function with exponent $\xi$. An analysis is made of the scaling of the steady second-order moments of the solenoidal field in two dimensions. The scaling exponent $\zeta_{l}$ of the isotropic part $(l=0)$ and the anisotropic part for the angular wave number $l=2$ is obtained analytically or numerically. The scaling of the isotropic part does not depend on whether the pressurelike term is present or not while the scaling of the anisotropic part is affected by the pressurelike term. There are two homogeneous similarity solutions with real positive exponents $\zeta_{2}$ when $\xi>\xi_{2}^{c} \approx 1.3$. The same kind of analysis is also applied to a simplified two-point closure equation.
\end{abstract}

DOI: 10.1103/PhysRevE.63.016308

PACS number(s): 47.27.Gs

\section{INTRODUCTION}

Turbulent flows in nature and technology are in general not isotropic due to the anisotropy of initial and boundary conditions, external forcing, etc. Although the flows cannot therefore be isotropic in a strict sense in particular at large scale, it has been widely accepted that the degree of the anisotropy in a statistical sense decreases with the scale in fully developed turbulence at high Reynolds number. If it is true, however, little seems to be known regarding how fast or slow the anisotropy decays with the scale, in spite of some pioneering studies (see, for example, Nelkin and Nakano [1], Arad et al. [2,3], and references cited therein). In this paper we consider this problem on the basis of a model of a solenoidal vector field that is advected by another rapidly changing random velocity field under the influence of a nonlocal effect which plays a role similar to the pressure in the Navier-Stokes dynamics and keeps the field incompressible.

The study of this model is motivated by the comparison between the equation for a randomly advected passive scalar field,

$$
\frac{\partial}{\partial t} \psi+(\mathbf{v} \cdot \nabla) \psi-\kappa \nabla^{2} \psi=f_{S},
$$

and the Navier-Stokes equation with the incompressibility condition,

$$
\begin{gathered}
\frac{\partial}{\partial t} \mathbf{u}+(\mathbf{u} \cdot \nabla) \mathbf{u}-\nu \nabla^{2} \mathbf{u}=-\nabla p+\mathbf{f}, \\
\boldsymbol{\nabla} \cdot \mathbf{u}=0,
\end{gathered}
$$

where $\mathbf{v}=\mathbf{v}(\mathbf{x}, t)$ is the random velocity field advecting the passive scalar $\psi=\psi(\mathbf{x}, t), \mathbf{u}=\mathbf{u}(\mathbf{x}, t)$ is the velocity of a fluid of unity density, $p=p(\mathbf{x}, t)$ is the pressure, $\kappa$ is the molecular diffusivity, $\nu$ is the kinematic viscosity, and $f_{S}$ and $\mathbf{f}$ are the external source and forcing, respectively.
One of the characteristic features of turbulence is the existence of the fluid motion and the advection effect associated with the motion. This effect is in fact included in Eq. (1), and is represented by the advection term $(\mathbf{v} \cdot \boldsymbol{\nabla}) \ldots$. Moreover, as shown by Kraichnan [4,5], if the characteristic time scale of $\mathbf{v}$ is very small and therefore may be assumed to be white in time, then Eq. (1) yields exact closure equations for multipoint moments such as $\left\langle\psi\left(\mathbf{x}_{1}, t\right) \psi\left(\mathbf{x}_{2}, t\right) \ldots\right\rangle$. Such exact closure equations are rare in the study of turbulence, and this model (hereafter called Kraichanan's model) of Eq. (1) with assuming the whiteness of $\mathbf{v}$ has stimulated extensive studies on the anomalous scaling of the moments $\left\langle[\psi(\mathbf{x}+\mathbf{r}, t)-\psi(\mathbf{x}, t)]^{n}\right\rangle$ for $n=2,3,4, \ldots$ (see, for example, Refs. [6-11], and references cited therein). The model is also expected to give some insight on the anomalous scaling of the velocity field obeying the Navier-Stokes equation.

On the other hand, it is also clear that there are differences between the Kraichnan's model (KM) for the passive scalar and the Navier-Stokes dynamics (NS). Among the differences are the following.

(a) The field $\psi$ in $\mathrm{KM}$ is a scalar, whereas the field $\mathbf{u}$ in NS is a vector satisfying the solenoidal condition (3).

(b) The pressure term in NS is absent in KM. Therefore, the evolution of $\psi$ in $\mathrm{KM}$ is local, whereas that of $\mathbf{u}$ in NS is nonlocal in the sense that the former at a point $\mathbf{x}$ is determined by the value of $\mathbf{v}$ and the spatial derivatives of the field itself (i.e., $\psi)$ at the point, whereas the latter is affected not only by the values at the point but also by the entire field of $\mathbf{u}$. [Note that Eqs. (2) and (3) give

$$
p=-\nabla^{-2}\left(\partial_{j} u_{k}\right)\left(\partial_{k} u_{j}\right),
$$

and $\nabla p$ in Eq. (2) is therefore affected by the entire velocity field $\mathbf{u}$, where $\nabla^{-2}$ is the integral operator representing the inverse of the Laplace operator $\nabla^{2}$, and we use the summation convention for repeated indices.]

(c) The convection velocity in $(\mathbf{u} \cdot \boldsymbol{\nabla})$ in NS is not white in time, in contrast to that in KM. 
(d) The dynamics of NS is nonlinear in $\mathbf{u}$, but $\mathrm{KM}$ as well as Eq. (1) is linear in $\psi$.

It would be certainly interesting to consider a model that may capture all the features of the NS dynamics listed above. However, at the present stage of our knowledge, it is difficult to construct a model that allows us to derive exact closure equations, but still represents the features of the NS dynamics not only in (a) and (b), but also (c) or (d). It may therefore be interesting to consider a model that may yield exact closures and also capture the features of the NS dynamics noted in (a) and (b). In this paper, we propose such a model in Sec. II, and analyze the scaling of the second-order moments of the model fields in two dimensions (2D) both for the isotropic part and the anisotropic part in Sec. III.

It may be worthwhile to recall here that Lagrangian twopoint closures such as the abridged Lagrangian history closure approximation (ALHDIA) [12] and the Lagrangian renormalized approximation (LRA) [13] are known to yield reasonable approximations that are free from any ad hoc adjusting parameter and in good agreement with experiments for the second-order moments of homogeneous and isotropic turbulence at high Reynolds number. They are applicable, at least in principle, also to anisotropic turbulence. It may be interesting, therefore, to apply such closures for the analysis of the dependence of anisotropy on the scale or the wave number. The analysis would, however, be very complicated because of the complexity of the closure equations. As will be shown in Sec. IV, the model presented in Sec. II yields a closure equation for the second-order moments that has a close relation to the one derived by a simplification of such two-point closure equations. This is another motivation of our studying the model.

\section{MODEL AND CLOSURE EQUATIONS FOR SECOND-ORDER MOMENTS}

One of the simple models capturing (a) and (b) may be formally obtained by a linearization of the Navier-Stokes equation with respect to the perturbation $(\widetilde{\mathbf{u}}, \widetilde{p}, \widetilde{\mathbf{f}})$ to a given basic stochastic state $\left(\mathbf{v}, p_{v}, \mathbf{f}_{v}\right)$. Substituting

$$
\mathbf{u}=\mathbf{v}+\widetilde{\mathbf{u}}, \quad p=p_{v}+\widetilde{p}, \quad \mathbf{f}=\mathbf{f}_{v}+\widetilde{\mathbf{f}},
$$

into Eq. (2), assuming that the basic state satisfies the Navier-Stokes equations (2) and (3), and retaining only terms linear in the perturbation, give

$$
\begin{gathered}
\frac{\partial}{\partial t} \widetilde{\mathbf{u}}+(\mathbf{v} \cdot \boldsymbol{\nabla}) \widetilde{\mathbf{u}}-\nu \Delta \widetilde{\mathbf{u}}=-\alpha(\widetilde{\mathbf{u}} \cdot \boldsymbol{\nabla}) \mathbf{v}-\beta \nabla \widetilde{\nabla}+\widetilde{\mathbf{f}}, \\
\boldsymbol{\nabla} \cdot \widetilde{\mathbf{u}}=0,
\end{gathered}
$$

where $(\alpha, \beta)=(1,1)$. In the model considered below, we further assume that the velocity $\mathbf{v}$ is white in time. The model then yields closure equations for multipoint correlations that are exact as in Kraichnan's model.

The left-hand-side of Eq. (4) is essentially similar to that of Eq. (1) except the fact that $\widetilde{\mathbf{u}}$ in the former is a vector, whereas $\psi$ in the latter is a scalar. If we set $(\alpha, \beta)=(0,0)$ and disregard Eq. (5), each component of the vector $\widetilde{\mathbf{u}}$ does not interact with the others and behaves as the passive scalar in Eq. (1). Studies have been made of the anomalous scaling of anisotropy of the passive scalar model in $[9,14]$. If we set $(\alpha, \beta)=(-1,0)$, then the model with the solenoidal condition (5) reduces to the one for a passive magnetic field, the anomalous scaling of which has been studied for both the isotropic case [15] and the anisotropic case [16]. Thus the model (4) includes both the models for a passive vector fields and for a passive magnetic field under appropriate choices of the values for the parameters $\alpha$ and $\beta$. Another interesting case may be the model (4) with $(\alpha, \beta)=(0,1)$. This model is simpler than the model with $(\alpha, \beta)=(1,1)$ in the sense that the stretching term $(\widetilde{\mathbf{u}} \cdot \boldsymbol{\nabla}) \mathbf{v}$ is absent, but still keeps the features of the Navier-Stokes dynamics noted in (a) and (b).

In the followings, we will consider the model with $(\alpha, \beta)=(1,1)$. The key feature of the model (4) lies in the presence of the nonlocal effect represented by the pressurelike term $\nabla \widetilde{p}$, by which the field $\widetilde{\mathbf{u}}$ is kept to be solenoidal. Taking the divergence of Eq. (4) and using Eq. (5) (with $\beta$ =1) give

$$
\nabla^{2} \widetilde{p}=-(1+\alpha)\left(\partial_{j} v_{k}\right)\left(\partial_{k} \widetilde{u}_{j}\right)
$$

so that

$$
\widetilde{p}(\mathbf{x})=-(1+\alpha) \int d \mathbf{y} G^{D}(\mathbf{x}-\mathbf{y})\left[\partial_{j} v_{k}(\mathbf{y})\right]\left[\partial_{k} \widetilde{u}_{j}(\mathbf{y})\right],
$$

where $D$ denotes the space dimension, and $G^{D}(\mathbf{x})$ is the Green function satisfying

$$
\nabla^{2} G^{D}(\mathbf{x})=\delta^{D}(\mathbf{x}),
$$

and appropriate boundary conditions, in which $\delta^{D}$ is the $D$-dimensional delta function. The parameter $\alpha$ is here written explicitly for later use although it is unity. In general, it is necessary to add to Eq. (6) integrals representing the contribution from $\boldsymbol{\nabla} \cdot \tilde{\mathbf{f}}$ and the boundary of the fluid domain. But for the sake of simplicity, we assume in this paper that the fields $\mathbf{v}, \widetilde{\mathbf{u}}, \widetilde{\mathbf{f}}$, and $\widetilde{p}$ satisfy the periodic boundary conditions and $\boldsymbol{\nabla} \cdot \widetilde{\mathbf{f}}=0$, so that we need not consider such a contribution. The integral in Eq. (6) is therefore to be understood as the one over the fundamental periodic domain.

By the use of Eq. (6), we may rewrite Eq. (4) as

$$
\frac{\partial}{\partial t} \widetilde{u}_{i}(\mathbf{x})=2 \int d \mathbf{y} M_{i j k}(\mathbf{x}, \mathbf{y}) v_{j}(\mathbf{y}) \widetilde{u}_{k}(\mathbf{y})+\nu \nabla^{2} \widetilde{u}_{i}(\mathbf{x})+\widetilde{f}_{i}(\mathbf{x}),
$$

where

$$
\begin{aligned}
M_{i j k}(\mathbf{x}, \mathbf{y})= & -\frac{1}{2} \delta^{D}(\mathbf{x}-\mathbf{y}) \delta_{i k} \partial_{j}^{\mathbf{y}}-\frac{\alpha}{2} \delta^{D}(\mathbf{x}-\mathbf{y}) \delta_{i j} \partial_{k}^{\mathbf{y}} \\
& +\gamma G^{D}(\mathbf{x}-\mathbf{y}) \partial_{i}^{\mathbf{y}} \partial_{j}^{\mathbf{y}} \partial_{k}^{\mathbf{y}},
\end{aligned}
$$


and $\gamma=(1+\alpha) / 2=1$. We will keep writing $\alpha$ and $\gamma$ explicitly (although they are unity) so that the contribution of the each term in Eq. (8) will be seen clearly.

Let the random advecting velocity $\mathbf{v}$ be statistically homogeneous in space and white noise process in time with zero mean and the correlation

$$
\int_{-\infty}^{0} d \tau\left\langle v_{i}(\mathbf{x}+\mathbf{r}, t) v_{j}(\mathbf{x}, t+\tau)\right\rangle=V_{i j}(\mathbf{r}, t)
$$

Then after some algebra it is shown that the single time second-order moment $U_{i j}(\mathbf{r}, t)$ defined by

$$
U_{i j}(\mathbf{r}, t)=\left\langle\tilde{u}_{i}(\mathbf{x}+\mathbf{r}, t) \tilde{u}_{j}(\mathbf{x}, t)\right\rangle,
$$

satisfies

$$
\begin{aligned}
\frac{\partial}{\partial t} U_{i j}(\mathbf{r}, t)= & \left\{L_{i j}[U]+\nu \nabla^{2} U_{i j}(\mathbf{r}, t)+F_{i j}(\mathbf{r})\right\} \\
& +\{(\mathbf{r}, i) \leftrightarrow(-\mathbf{r}, j)\},
\end{aligned}
$$

where $L_{i j}$ is the linear operator defined by

$$
\begin{aligned}
L_{i j}[U]= & 4 \int d \mathbf{x} \int d \mathbf{y}\left[M_{i a b}(\mathbf{r}, \mathbf{x}) M_{j c d}(\mathbf{0}, \mathbf{y})\right. \\
& \times V_{a c}(\mathbf{x}-\mathbf{y}, t) U_{b d}(\mathbf{x}-\mathbf{y}, t)+M_{i a b}(\mathbf{r}, \mathbf{x}) M_{b c d}(\mathbf{x}, \mathbf{y}) \\
& \left.\times V_{a c}(\mathbf{x}-\mathbf{y}, t) U_{d j}(\mathbf{y}, t)\right]
\end{aligned}
$$

and the random force $\tilde{\mathbf{f}}$ is assumed to be statistically stationary and independent from $\mathbf{v}$ and $\widetilde{\mathbf{u}}$ with zero mean and

$$
F_{i j}(\mathbf{r})=\int_{-\infty}^{0} d \tau\left\langle\widetilde{f}_{i}(\mathbf{x}+\mathbf{r}, t) \widetilde{f}_{j}(\mathbf{x}, t+\tau)\right\rangle
$$

The expression $L_{i j}[U]$ may be simplified by substituting the expression (8) of $M_{i j k}$ into (10). In particular, the trace $L_{i i}[U]$ may be then written as

$$
\begin{aligned}
L_{i i}[U]= & {\left[V_{a c}(\mathbf{0}, t)-V_{a c}(\mathbf{r}, t)\right] \partial_{a} \partial_{c} U_{i i}(\mathbf{r}, t)-\alpha\left[\partial_{b} V_{i c}(\mathbf{r}, t)\right] } \\
& \times\left[\partial_{c} U_{b i}(\mathbf{r}, t)\right]-\alpha\left[\partial_{d} V_{a i}(\mathbf{r}, t)\right]\left[\partial_{a} U_{i d}(\mathbf{r}, t)\right] \\
& -\alpha^{2}\left[\partial_{b} \partial_{d} V_{i i}(\mathbf{r}, t)\right] U_{b d}(\mathbf{r}, t) \\
& +\int d \mathbf{p}\left\{4 \gamma(1+\alpha-\gamma) G^{D}(\mathbf{q}, t)\left[\partial_{b} \partial_{d} V_{a c}(\mathbf{p}, t)\right]\right. \\
& \times\left[\partial_{a} \partial_{c} U_{b d}(\mathbf{p}, t)\right]-2 \gamma\left[\partial_{a} \partial_{c} \partial_{d} \partial_{i} G^{D}(\mathbf{q})\right] \\
& \times V_{a c}(\mathbf{q}, t) U_{d i}(\mathbf{p}, t)+2 \alpha \gamma\left[\partial_{a} \partial_{c} G^{D}(\mathbf{q})\right] \\
& \left.\times\left[\partial_{a} \partial_{d} V_{i c}(\mathbf{q}, t)\right] U_{d i}(\mathbf{p}, t)\right\},
\end{aligned}
$$

where $\mathbf{q}=\mathbf{r}-\mathbf{p}$. Now we set the sides of the fundamental domain to infinity. Then integrals in Eq. (12) are to be taken over $\mathbf{R}^{D}$

In the following, we assume that the random advecting field $\mathbf{v}$ is statistically stationary, homogeneous, and isotropic with zero mean, and has a scaling range of $r$ such that

$$
\eta \ll r=|\mathbf{r}| \ll L
$$

in which the second-order moment of the increment $\delta v_{i}(\mathbf{r}, t)=\left[v_{i}(\mathbf{r}, t)-v_{i}(\mathbf{0}, t)\right]$ is given by

$$
\begin{aligned}
\int_{-\infty}^{0} d \tau\left\langle\delta v_{i}(\mathbf{r}, t) \delta v_{j}(\mathbf{r}, t+\tau)\right\rangle & =2\left[V_{i j}(\mathbf{0}, t)-V_{i j}(\mathbf{r}, t)\right] \\
& =2 r^{\xi}\left(\delta_{i j}-\frac{\xi}{D-1+\xi} \frac{r_{i} r_{j}}{r^{2}}\right),
\end{aligned}
$$

with

$$
0<\xi<2 \text {. }
$$

Here $\eta$ is the characteristic length scale of the viscous subrange, while $L$ is that of energy containing eddies of $\mathbf{v}$ as well as $\widetilde{\mathbf{u}}$ and the forcing $\widetilde{\mathbf{f}}$, so that $F_{i j}(\mathbf{r})$ is almost constant, say $C_{i j}$, independent of $\mathbf{r}$ in the scaling range (13). We assume that $F_{i j}$ is almost isotropic so that $C_{i j}=C \delta_{i j}$.

We further assume that there is a (quasi)stationary state in which the time dependence of $U_{i j}(\mathbf{r}, t)$ is negligible in the scaling range (13). The assumption is acceptable when the correlation function $U_{i j}(\mathbf{r}, t)$ for the force-free case decays with time and therefore unbounded growth of the field (dynamo effect) does not occur. If the dynamo effect does not occur, the stationary state may be achieved by a large-scalecorrelated external forcing f. Note that from the CauchySchwartz inequality, the absolute value of the correlation function $\left|U_{i j}(\mathbf{r}, t)\right|$ at any $\mathbf{r}$ is bounded by the value of the trace $\left|U_{i i}(\mathbf{0}, t)\right|$ at $\mathbf{r}=\mathbf{0}$ which is proportional to the energy of the field per unit mass.

In the model with $(\alpha, \beta)=(0,0)$ (which is essentially the same as the passive scalar model), the right-hand-side of Eq. (12) vanishes at $\mathbf{r}=\mathbf{0}$ and therefore $U_{i i}(\mathbf{0}, t)$ decays with time due to the viscosity, which implies that there is no dynamo effect. In the model with $(\alpha, \beta)=(0,1)$ (the model of passive solenoidal vector without stretching), the absence of dynamo is shown in the same way. In the model with $(\alpha, \beta)=$ $(-1,0)$ (the passive magnetic field model), it is shown by Vergassola that there is no dynamo effect in 2D for any exponent $\xi$, and in 3D for $0<\xi<1$ [15].

The question whether the dynamo effect occurs or not in the model with $(\alpha, \beta)=(1,1)$ is not yet solved. In the forcefree case, we may rewrite Eq. (9) for the trace of correlation function $U_{i i}(\mathbf{r}, t)$ as

$$
\begin{aligned}
\frac{\partial}{\partial t} U_{i i}(\mathbf{r}, t)= & 2\left\{\left.L_{i i}[U](\mathbf{r}, t)\right|_{(\alpha, \beta)=(1,1)}+\nu \nabla^{2} U_{i i}(\mathbf{r}, t)\right\} \\
= & 2 \frac{\left\{\left.L_{i i}[U](\mathbf{r}, t)\right|_{(\alpha, \beta)=(-1,0)}+\nu \nabla^{2} U_{i i}(\mathbf{r}, t)\right.}{} \\
& \left.+L_{i i}^{\prime}[U](\mathbf{r}, t)\right\},
\end{aligned}
$$

where the operator $L_{i i}^{\prime}[\cdot]$ is defined by $\left.L_{i i}[\cdot]\right|_{(\alpha, \beta)=(1,1)}$ $-\left.L_{i i}[\cdot]\right|_{(\alpha, \beta)=(-1,0)}$. If the term $L_{i i}^{\prime}[U]$ is absent, Eq. $(15)$ is identical to the equation for the model with $(\alpha, \beta)=$ $(-1,0)$ and there is no dynamo effect in the parameter re- 
gion noted above. If the underlined part of Eq. (15) is absent, it can be shown after some algebra that $U_{i i}(\mathbf{0}, t)$ $=\sup _{\mathbf{r}}\left|U_{i i}(\mathbf{r}, t)\right|$ decays for $D=2,3$, and $0<\xi<2$, therefore no dynamo effect occurs. However, the absence of dynamo in the full system (15) is not in general guaranteed by the fact that each of the two separated parts in the right-handside of Eq. (15) does not induce the dynamo effect when the other is absent. The difficulty of the problem lies in the facts that $L_{i i}[U](\mathbf{0}, t)$ is non-negative for any correlation function $U_{i j}(\mathbf{r}, t)$ if the stretching term is present $(\alpha=1)$ and that the operator $L_{i j}[\cdot]$ contains not only derivatives but also integrals (in space) due to the pressurelike term $(\beta=1)$.

If $U_{i j}(\mathbf{r}, t)$ is the correlation function of the stationary state, then

$$
L_{i j}[U]=-C \delta_{i j}
$$

Since $L$ is a linear operator, the solution of Eq. (16) may be symbolically written as

$$
U=U^{H}+U^{I}
$$

where $U^{H}$ and $U^{I}$ are the homogeneous and inhomogeneous solutions of Eq. (16), respectively. The determination of the scaling of the solution of Eq. (16) requires the knowledge of the scaling not only of the inhomogeneous solution but also those of homogeneous solutions.

Regarding the former, it is readily shown by a power counting that the isotropic inhomogeneous solution whose second-order moment scales as

$$
U_{i j}^{I}(\mathbf{r}) \propto r^{\zeta_{I}}
$$

has the scaling exponent $\zeta_{I}=2-\xi$. Here, we have omitted writing the time argument for we are considering the stationary solution in the scaling range (13).

However, such a power-counting method or dimensional consideration is insufficient to determine the scaling of the homogeneous solutions. Its determination requires an analysis (the so-called zero-mode analysis) of the homogeneous equation. Although the analysis in 3D would be quite complicated, the analysis can be considerably simplified in $2 \mathrm{D}$ because the second-order tensor $U_{i j}$ may be then expressed in terms of only one scalar function. As a first step toward the understanding on the scaling implied in the homogeneous equation, we therefore consider the scaling in 2D in Sec. III.

\section{ANOMALOUS SCALING OF SECOND-ORDER MOMENTS IN 2D}

\section{A. Formulation for 2D}

By virtue of the incompressibility condition, the correlation $U_{i j}$ in $2 \mathrm{D}$ may be expressed in terms of a scalar function, say $\Phi$, as

$$
U_{i j}(\mathbf{r})=\epsilon_{i a} \epsilon_{j b} \partial_{a} \partial_{b} \Phi(\mathbf{r})
$$

where $\epsilon_{12}=-\epsilon_{21}=1, \epsilon_{11}=\epsilon_{22}=0$, and $\Phi(-\mathbf{r})=\Phi(\mathbf{r})$.

Since $L$ is a linear operator, the homogeneous solution may be expressed as a linear combination of the functions of the form

$$
U_{i j}^{l}(\mathbf{r})=\epsilon_{i a} \epsilon_{j b} \partial_{a} \partial_{b} \Phi_{l}(\mathbf{r})
$$

where

$$
L_{i j}\left[U^{l}\right]=0
$$

and

$$
\Phi_{0}(\mathbf{r})=R_{0}(r)
$$

$$
\Phi_{l}(\mathbf{r})=R_{l}^{c}(r) \cos (l \theta)+R_{l}^{s}(r) \sin (l \theta) \quad(\text { when } l \neq 0) .
$$

The correlation function $U_{i j}(\mathbf{r})$ satisfies $U_{i j}(-\mathbf{r})=U_{i j}(\mathbf{r})$ in $2 \mathrm{D}$ so that $l$ is even. The isotropic part of $U_{i j}(\mathbf{r})$ is given by $U_{i j}^{0}(\mathbf{r})$. Since the scaling behaviors of $R_{l}^{c}(r)$ and $R_{l}^{s}(r)$ are the same, we discuss only the cosine part. We let $R_{l}^{s}(r)=0$ and denote $R_{l}^{c}(r)$ by $R_{l}(r)$ in what follows. From Eqs. (12) and (14), Eq. (18) can be written in terms of $R_{l}(r)$ as

$$
\begin{aligned}
L_{i i}\left[U^{l}\right]= & \frac{1}{1+\xi}\left(r ^ { \xi - 4 } \operatorname { c o s } ( l \theta ) \left(\left\{r^{4} R_{l}^{\prime \prime \prime \prime}(r)+(2+\xi) r^{3} R_{l}^{\prime \prime \prime}(r)+\left[\xi-1-l^{2}(2+\xi)\right] r^{2} R_{l}^{\prime \prime}(r)+\left(1+2 l^{2}\right)(1-\xi) r R_{l}^{\prime}(r)\right.\right.\right. \\
& \left.+\left[2 l^{2}(\xi-2)+l^{4}(1+\xi)\right] R_{l}(r)\right\}+2 \alpha\left[-\xi r^{3} R_{l}^{\prime \prime \prime}(r)+\xi(1-\xi) r^{2} R_{l}^{\prime \prime}(r)+\xi\left(1+l^{2}\right)(\xi-1) r R_{l}^{\prime}(r)\right. \\
& \left.\left.+2 l^{2} \xi(1-\xi) R_{l}(r)\right]+\alpha^{2} \xi(\xi+2)\left[r^{2} R_{l}^{\prime \prime}(r)+(\xi-1) r R_{l}^{\prime}(r)+l^{2}(1-\xi) R_{l}(r)\right]\right)+2 \gamma(1+\alpha-\gamma) \xi \\
& \times \int_{\mathbf{R}^{2}} d \mathbf{p} \frac{p^{\xi-6} \ln (q)}{\pi} \cos \left(l \theta_{r p}\right)\left\{-3 p^{4} R_{l}^{\prime \prime \prime \prime}(p)+6(1-\xi) p^{3} R_{l}^{\prime \prime \prime}(p)+3(1-\xi)\left(\xi-2 l^{2}-3\right) p^{2} R_{l}^{\prime \prime}(p)\right. \\
& \left.+3\left(1+2 l^{2}\right)(3-\xi)(1-\xi) p R_{l}^{\prime}(p)+\left[l^{4}(\xi+1)+l^{2}(8 \xi-28)\right](1-\xi) R_{l}(p)\right\}+2 \gamma[3+(\xi-1) \alpha] \xi \int_{\mathbf{R}^{2}} d \mathbf{p} \frac{p^{-2} q^{\xi-4}}{\pi} \\
& \left.\times\left\{\cos \left(2 \theta_{p q}\right) \cos \left(l \theta_{r p}\right)\left[-p^{2} R_{l}^{\prime \prime}(p)+p R_{l}^{\prime}(p)-l^{2} R_{l}(p)\right]+2 l \sin \left(2 \theta_{p q}\right) \sin \left(l \theta_{r p}\right)\left[p R_{l}^{\prime}(p)-R_{l}(p)\right]\right\}\right)=0,
\end{aligned}
$$


where $\mathbf{q}=\mathbf{r}-\mathbf{p}, \theta_{r p}$ and $\theta_{p q}$ denote the angles between two vectors $\mathbf{r}, \mathbf{p}$ and $\mathbf{p}, \mathbf{q}$, respectively, and the prime denotes the derivative of the function. The tensorial algebra was performed by using MATHEMATICA.

Let $R_{l}$ be given by

$$
R_{l}(r)=r^{2+\zeta}
$$

in the scaling range (13), then $U_{i j}^{l}(\mathbf{r})$ is given by

$$
\begin{aligned}
U_{i j}^{l}(\mathbf{r})= & r^{\zeta}\left[\cos (l \theta)\left((\zeta+2)(\zeta+1) \delta_{i j}-\left[(\zeta+2) \zeta+l^{2}\right] \frac{r_{i} r_{j}}{r^{2}}\right)\right. \\
& \left.-\sin (l \theta) l(\zeta+1)\left(\epsilon_{i a} \frac{r_{a} r_{j}}{r^{2}}+\epsilon_{j a} \frac{r_{a} r_{i}}{r^{2}}\right)\right]
\end{aligned}
$$

From Eqs. (19) and (20), Eq. (18) reduces to

$$
L_{i i}\left[U^{l}\right]=\lambda_{\xi, l}(\zeta) r^{\xi+\zeta-2} \cos (l \theta)=0
$$

i.e.,

$$
\lambda_{\xi, l}(\zeta)=0
$$

where

$$
\begin{aligned}
\lambda_{\xi, l}(\zeta)= & \frac{1}{1+\xi}\left(\left[(2+\zeta)^{2}-l^{2}\right]\left[\zeta(\zeta+\xi)-l^{2}(1+\xi)\right]+\right. \\
& {\left[-2 \alpha \zeta \xi+\alpha^{2} \xi(2+\xi)\right]\left[(2+\zeta)(\zeta+\xi)+l^{2}(1-\xi)\right] } \\
& +\frac{4 \gamma(1+\alpha-\gamma) \xi}{l^{2}-(\zeta+\xi-2)^{2}}[3 \zeta(2+\zeta)(\xi+\zeta)(\xi+\zeta-2) \\
& \left.+2 l^{2}(1-\xi)\left(2+3 \zeta^{2}+2 \xi+3 \zeta \xi\right)+l^{4}\left(\xi^{2}-1\right)\right] \\
& -2 \gamma[(\xi-1) \alpha+3] \xi \int_{\mathbf{R}^{2}} d \mathbf{p} \frac{p^{\zeta} q^{-4+\xi}}{\pi} \\
& \times\left\{\left[l^{2}+\zeta(2+\zeta)\right] \cos \left(l \theta_{r p}\right) \cos \left(2 \theta_{p q}\right)\right. \\
& \left.\left.-2 l(1+\zeta) \sin \left(l \theta_{r p}\right) \sin \left(2 \theta_{p q}\right)\right\}\right),
\end{aligned}
$$

and $\mathbf{q}=\mathbf{r} / r-\mathbf{p}$. The integral on the right-hand side of Eq. (23) converges when $\xi>0$ and $-2-|l-2|<\zeta<2-\xi+l$.

Therefore the scaling of the homogeneous solution is given by

$$
U_{i j}^{l}(\mathbf{r}) \propto r^{\zeta_{l}}
$$

where $\zeta_{l}$ is the solutions of Eq. (22). Although $\zeta_{l}$ depends on $\xi$, we do not write it here explicitly. Because of the complexity of the integral, it is not easy to solve Eq. (22) analytically. However, it can be solved numerically. In what follows, integrals in computations are evaluated numerically by using MATHEMATICA.

\section{B. Scaling of the isotropic part}

For the model with $(\alpha, \beta)=(1,1)$, it is shown analytically that $\zeta_{0}=-\xi$ is a solution of Eq. (22) as follows. For $l=0$ and $\zeta=-\xi$, the integral in Eq. (23) is proportional to

$$
\int_{\mathbf{R}} d \mathbf{p} \frac{1}{q^{4}}\left(\frac{q}{p}\right)^{\zeta} \cos \left(2 \theta_{p q}\right) .
$$

By introducing variables $\left(\rho, \kappa, \theta_{\rho}\right)$ defined as

$$
\rho=\frac{p}{q}, \quad \kappa=\frac{1}{q}, \quad \theta_{\rho}=\theta_{p q},
$$

and noting that the triangle with the sides of length $1, \rho$, and $\kappa$ is similar to that with the length $q, p$, and 1 , the integral (24) is shown to be

$$
\int_{0}^{\infty} d \rho \int_{0}^{2 \pi} d \theta_{\rho} \rho^{-\xi+1} \cos \left(2 \theta_{\rho}\right)=0 .
$$

The other part of the right-hand-side of Eq. (23) also vanishes when $l=0$ and $\zeta=-\xi$. Therefore, $\zeta_{0}=-\xi$ is a solution of Eq. (22). The value $\lambda_{\xi, 0}(\zeta)$ defined by Eq. (23) may be evaluated numerically in the parameter region of $\xi>0$ and $-4<\zeta<2-\zeta$ where the integral in Eq. (23) converges. It is then found that there is a negative solution $\zeta_{0}^{(2)}$ of Eq. (22) other than $\zeta_{0}=-\xi$ whose absolute value is larger than $\xi$. Therefore, the homogeneous equation (18) has a solution in the scaling range (13) whose trace is given by

$$
U_{i i}^{H}(\mathbf{r})=A_{1} r^{\zeta_{0}^{(1)}}+A_{2} r^{\zeta_{0}^{(2)}}
$$

where $A_{1}$ and $A_{2}$ are arbitrary constants and $\zeta_{0}^{(1)}=-\xi$. Now assume that the trace of the correlation function $U_{i i}^{H}(\mathbf{r})$ is of the form (25) in the scaling range (13) and that the two terms in the right-hand-side of Eq. (25) are of the same order at the small scale $\eta$, that is, $A_{2} / A_{1} \sim \eta^{\zeta_{0}^{(1)}}-\zeta_{0}^{(2)}$. Then the term proportional to $r^{\zeta_{0}^{(1)}}$ is much larger than the term proportional to $r^{\zeta_{0}^{(2)}}$ in the scaling range (13) and therefore the dominant scaling behavior is $U_{i i}^{H} \propto r^{\zeta_{0}^{(1)}}=r^{-\xi}$. As mentioned in Sec. II, the inhomogeneous solution with scaling exponent $\zeta_{I}=2$ $-\xi$ is to be added to the homogeneous solution given above.

For later use, we extend the assumption to determine the dominant scaling behavior in the scaling range (13) to the case when there are more than two isotropic homogeneous solutions of Eq. (18) with different exponents $0 \geqslant \zeta_{0}^{(1)}>\zeta_{0}^{(2)}$ $>\ldots$. We assume that the homogeneous part of the correlation function $U^{H}$ is expressed as a linear combination of these scaling solutions of Eq. (18) in the scaling range (13) and that all the scaling solution are of the same order at $r$ $\sim \eta$. It follows that the dominant scaling behavior in the scaling range is determined by the largest exponent $\zeta_{0}^{(1)}$.

The scaling exponents of the isotropic second-order moment in the model with other value of the parameters $(\alpha, \beta)$ are given in the following for the comparison. In the rest of this section, $\zeta_{l}(\alpha, \beta)$ denotes the scaling exponent of the homogeneous equation (18) with $(\alpha, \beta)$. The model with 
$(\alpha, \beta)=(0,0)$ has the same scaling exponent as that of the passive scalar model. Although the solenoidal condition (5) is not applied for the model with $(\alpha, \beta)=(0,0)$, it can be shown that we can formally apply Eqs. (22) and (23) to determine the scaling exponent $\zeta_{0}$ by letting $(\alpha, \gamma)=(0,0)$ and eliminating the zero's coming from the coefficient $[(2$ $\left.+\zeta)^{2}-l^{2}\right]$. The exponents are $\zeta_{0}(0,0)=0,-\xi$ and from the assumption mentioned above, the exponent of the dominant scaling is given by $\zeta_{0}(0,0)=0$, that is, the homogeneous solution is nearly a constant. For the model with $(\alpha, \beta)=$ $(-1,0)$ (the passive magnetic field model), the scaling exponents of the homogeneous solutions are given by solving Eq. (22) with $\alpha=-1$ and $\gamma=0$. Among the exponents $\zeta_{0}$ $(-1,0)=-\xi,-2,-\xi,-2$, the largest exponent $\zeta_{0}(-1,0)=$ $-\xi$ dominates the scaling behavior in the scaling range (13) from the assumption. The exponent $\zeta_{0}(-1,0)=-\xi$ is consistent with the result in [15]. For the model with $(\alpha, \beta)$ $=(0,1)$, the scaling exponents of the homogeneous solutions are given by solving Eq. (22) with $\alpha=0$ and $\gamma=1 / 2$. It is easily seen that $\zeta_{0}(0,1)=0$ is a solution. In a similar way as for $(\alpha, \beta)=(1,1)$, it can be proved that $\zeta_{0}(0,1)=-\xi$ is a solution. It is found numerically that there exist negative exponents $\zeta_{0}(0,1)$ whose absolute values are larger than $\xi$ and there is no positive exponent in the parameter region of $\xi$ and $\zeta$ where the integral in Eq. (23) converges. Therefore the exponent of the dominant scaling behavior is determined to be $\zeta_{0}(0,1)=0$. Note that the inhomogeneous solution of Eq. (16) with the scaling exponent $\zeta_{I}=2-\xi$ is to be added to the homogeneous solutions in all the cases of $(\alpha, \beta)$ shown above.

The scaling exponent $\zeta_{0}(\alpha, \beta)$ of the homogeneous solution of Eq. (18) is 0 if the stretching term is absent $(\alpha=0)$ and $-\zeta$ if it is present $(\alpha= \pm 1)$ and $\beta$ is irrelevant to the exponent $\zeta_{0}(\alpha, \beta)$. Therefore the scaling of the isotropic part is determined by whether the stretching is present or not and is not affected by the pressurelike term.

\section{Scaling of the anisotropic part}

Since the constant term $C \delta_{i j}$ in Eq. (16) is zero except for $l=0$, we have only to consider the homogeneous equation (18). Equation (22) with $(\alpha, \beta)=(1,1)$ is solved numerically for $l=2$. The solution is shown in Fig. 1. It suggests that there is a critical value $\xi_{2}^{c}$ such that $\lambda_{\xi, 2}(\zeta)=0$ has no real zero for $\xi<\xi_{2}^{c}$, but has real zero's $\zeta_{2}^{(1)}(1,1)$ and $\zeta_{2}^{(2)}(1,1)$ such that $0<\zeta_{2}^{(1)}(1,1)<\zeta_{2}^{(2)}(1,1)<2$ for $\xi>\xi_{2}^{c}$, and the critical values is $\xi_{2}^{c} \approx 1.3$. Note that the correlation function $U_{i j}^{l}(\mathbf{r})$ for the anisotropic part $(l \neq 0)$ is 0 at $\mathbf{r}=\mathbf{0}$ and that the exponent $\zeta_{l}$ which matches the small scale limit is positive. If there is more than one scaling solution with positive exponents $\zeta_{l}$, then the one with the smallest exponent decays slowest with the scale $r$ and dominates the scaling in the scaling range (13). Therefore in the case for $l=2$, the exponent of the dominant scaling is $\zeta_{2}^{(1)}$. It is found for $l=4$ numerically that the two real zero's $2<\zeta_{4}^{(1)}(1,1)<\zeta_{4}^{(2)}(1,1)$ $<4$ of Eq. (22) which take nontrivial values exist for $\xi$ $>\xi_{4}^{c}$ where $\xi_{4}^{c}<1 / 3$. Therefore, the homogeneous solutions for $l=4$ decay with the scale faster than those for $l=2$. The

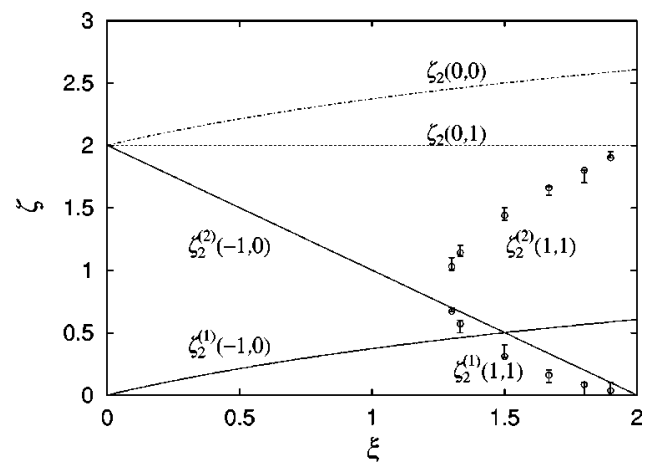

FIG. 1. The scaling exponents $\zeta_{2}$ of the homogeneous solutions of the model equation $[(\alpha, \beta)=(1,1)]$ with $l=2$. $\xi$ is the scaling exponent of the advecting field. $\lambda_{\xi, 2}(\xi)$ changes its sign within the error bars and the circle symbols denote zero's obtained by a linear interpolation. For comparison, the scaling exponents $\zeta_{2}$ of the model with other choices of the values of the parameters $(\alpha, \beta)$ are also given by the solid, dashed, and dot-dashed lines.

fact is in agreement with the empirical "law of isotropization," which states that the anisotropy of the higher degree $l$ decays faster with the scale.

The scaling exponents of the anisotropic part for the model with the other values of parameters $(\alpha, \beta)$ are given in the following for comparison. For the model with $(\alpha, \beta)$ $=(0,0)$, it can be shown that

$$
\zeta_{l}(0,0)=\frac{1}{2}\left[-\xi+\sqrt{\xi^{2}+4(1+\xi) l^{2}}\right],
$$

by using the same procedure as for the isotropic case to Eq. (23). Only the positive exponent that matches the small scale limit is shown. The exponent $\zeta_{l}(0,0)$ is the same as that of the passive scalar model which is given by Fairhall et al. [9]. The exponent $\zeta_{2}(0,0)$ is also plotted in Fig. 1. For the model with $(\alpha, \beta)=(-1,0)$ (the passive magnetic field model) the scaling exponents of the homogeneous solutions are given by solving Eq. (22) with $\alpha=-1$ and $\gamma=0$,

$$
\begin{gathered}
\zeta_{l}^{(1)}(-1,0)=\frac{1}{2}\left[-\xi+\sqrt{\xi^{2}+4(1+\xi) l^{2}}\right]-2, \\
\zeta_{l}^{(2)}(-1,0)=-\xi+l,
\end{gathered}
$$

where negative exponents are omitted for the same reason as for the case $(\alpha, \beta)=(0,0)$. The exponents are shown in Fig. 1 for $l=2$ for comparison. For the model with $(\alpha, \beta)$ $=(0,1)$, it is easily seen that $\zeta_{2}(0,1)=2$ is a solution of Eq. (23) for all $0<\xi<2$ as follows. The integrand in Eq. (23) is proportional to $p^{2} q^{-4+\xi} \cos \left(2 \theta_{r q}\right)$ when $\zeta=2$ and $l=2$. Since $p^{2}=1+q^{2}-2 q \cos \left(\theta_{r q}\right)$, the integral is shown to be 0 . In the case for $(\alpha, \beta)=(0,1)$, the other part of Eq. (23) also vanishes for $\zeta=2$ and $l=2$. Therefore $\zeta_{2}(0,1)=2$ is a solution of Eq. (22) for all $0<\xi<2$. It is shown numerically that there is no other positive solution $\zeta_{2}(0,1)$ of Eq. (22) in the range of $\xi$ and $\zeta$ where the integral in Eq. (23) converges. It is not easy to solve Eq. (22) analytically for $l \geqslant 4$, but it is 
shown numerically that there exist two zero's $2<\zeta_{4}^{(1)}(0,1)$ $<\zeta_{4}^{(2)}(0,1)<6-\xi$ of Eq. (22) with nontrivial values for $\xi$ $>\xi_{4}^{\prime c}$ where $\xi_{4}^{\prime c}<1 / 3$.

From the comparison of the models with different values of parameters $(\alpha, \beta)$, it is found that the scaling exponent $\zeta_{l}(\alpha, \beta)$ of the homogeneous solution of Eq. (18) is affected by the pressurelike term for the anisotropic part $(l \neq 0)$, while it is not the case for the isotropic part $(l=0)$. It is seen from Fig. 1 that $\zeta_{2}(0,1)<\zeta_{2}(0,0)$ and $\zeta_{2}^{(1)}(1,1)$ $<\zeta_{2}^{(2)}(-1,0)$ if $\zeta_{2}^{(1)}(1,1)$ exists, which suggests that the presence of the pressurelike term $(\beta=1)$ lowers the scaling exponent $\zeta_{2}$ of the anisotropic homogeneous solution of Eq. (18).

\section{A SIMPLIFICATION OF THE TWO-POINT CLOSURE EQUATION}

In various two-time two-point closure approximations including the direct interaction approximation (DIA), as well as the ALHDIA and the LRA, the evolution of a single time moment $U_{i j}(\mathbf{r}, t)$ of the fluid velocity in homogeneous turbulence obeying the Navier-Stokes equation (2) is given by the equation of the form

$$
\begin{aligned}
\frac{\partial}{\partial t} U_{i j}(\mathbf{r}, t)= & \int d \mathbf{x} d \mathbf{y} d \mathbf{z} \int_{t_{0}}^{t} d s\left\{2 M_{i a b}(\mathbf{r}, \mathbf{x}) M_{e c d}(\mathbf{z}, \mathbf{y})\right. \\
& \times Q_{a c}(\mathbf{x}-\mathbf{y} ; t, s) Q_{b d}(\mathbf{x}-\mathbf{y} ; t, s) G_{j e}(-\mathbf{z} ; t, s) \\
& +4 M_{i a b}(\mathbf{r}, \mathbf{x}) M_{d c e}(\mathbf{y}, \mathbf{z}) Q_{a c}(\mathbf{x}-\mathbf{y} ; t, s)
\end{aligned}
$$

$$
\left.\times G_{b d}(\mathbf{x}-\mathbf{y} ; t, s) Q_{e j}(\mathbf{z} ; t, s)\right\}+\{(\mathbf{r}, i) \leftrightarrow(-\mathbf{r}, j)\},
$$

where we have omitted writing the viscous and forcing term. $M_{i j k}(\mathbf{x}, \mathbf{y})$ is that of Eq. (8) with $\alpha=1$ and $\gamma=1$. The two time functions $Q_{i j}(\mathbf{r} ; t, s)$ and $G_{i j}(\mathbf{r} ; t, s)$ are to be understood as appropriately defined two-time correlation and response functions. For example, they are Eulerian two-time functions in the DIA, whereas they are Lagrangian functions in the ALHDIA and the LRA.

Let us suppose that the turbulence is weakly anisotropic, and we may write

$$
Q_{i j}(\mathbf{r} ; t, s)=V_{i j}(\mathbf{r} ; t, s)+U_{i j}(\mathbf{r} ; t, s)
$$

where $V_{i j}$ is the two-time two-point correlation for a certain isotropic state of turbulence, and $U_{i j}$ represents the perturbation from the isotropic state. By substituting Eq. (29) into Eq. (28) and collecting only the terms first order in $U_{i j}$, and further introducing a bold simplification that the characteristic time scale of $V$ is very small so that one may put $V_{i j}(\mathbf{r} ; t, s) \propto \delta(t-s)$, we obtain

$$
\frac{\partial}{\partial t} U_{i j}(\mathbf{r}, t)=\left\{L_{i j}[U]+4 \int d \mathbf{x} \int d \mathbf{y} M_{i a b}(\mathbf{r}, \mathbf{x}) M_{b c d}(\mathbf{x}, \mathbf{y}) U_{a c}(\mathbf{x}-\mathbf{y}, t) V_{d j}(\mathbf{y}, t)\right\}+\{(\mathbf{r}, i) \leftrightarrow(-\mathbf{r}, j)\},
$$

where

$$
V_{i j}(\mathbf{r} ; t)=\int_{-\infty}^{t} V_{i j}(\mathbf{r} ; t, s) d s, \quad U_{i j}(\mathbf{r}, t)=U_{i j}(\mathbf{r} ; t, t)
$$

Comparison between Eqs. (30) and (10) shows that the simplified two-point closure equation (30) and the equations derived by the model (4) have some similarity to each other. Both have the same operator $L$, and the only difference is the existence of the underlined extra term in Eq. (30).

The equation for the second-order moment is given by

$$
\begin{aligned}
\frac{\partial}{\partial t} U_{i i}(\mathbf{r})= & 2\left(L_{i i}[U]+2 \int d \mathbf{p}\left\{\left[\partial_{a} G(\mathbf{p})\right]\left[\partial_{b} \partial_{d} V_{a c}(\mathbf{q})\right]\right.\right. \\
& \times\left[\partial_{c} U_{b d}(\mathbf{p})\right]+\left[\partial_{a} G(\mathbf{p})\right]\left[\partial_{a} \partial_{d} V_{b c}(\mathbf{q})\right] \\
& \left.\left.\times\left[\partial_{c} U_{b d}(\mathbf{p})\right]\right\}\right)
\end{aligned}
$$

We assume the existence of a statistically stationary state for this equation, too. Now one can estimate in 2D the anomalous scaling in the range (13) in the same way as in Sec. III. In particular, if we put $V_{i j}$ as Eq. (14) and $U_{i j}^{l}$ as Eq. (21) then we have a relation for the homogeneous solution that may be written in the form similar to Eq. (22), say

$$
\lambda_{\xi, l}^{\prime}(\zeta)=0
$$

where

$$
\begin{aligned}
\lambda_{\xi, l}^{\prime}(\zeta)= & \lambda_{\xi, l}(\zeta)+\frac{1}{1+\xi} \int_{\mathbf{R}^{2}} d \mathbf{p} \frac{p^{-2+\zeta} q^{-2+\zeta}}{4 \pi} \xi\left[\zeta \left(\left[l^{2}\right.\right.\right. \\
& \left.-(2+\zeta)^{2}\right]\left\{\xi(2+\xi)+2\left(4-\xi^{2}\right) \cos \left(2 \theta_{p q}\right)\right\} \\
& \left.+\left(4-3 l^{2}-\zeta^{2}\right)\left(8-6 \xi+\xi^{2}\right) \cos \left(4 \theta_{p q}\right)\right) \cos \left(l \theta_{r p}\right) \\
& +l(\xi-2)\left\{2\left[l^{2}-(2+\zeta)^{2}\right](2+\zeta) \sin \left(2 \theta_{p q}\right)\right. \\
& \left.\left.+\left(4-l^{2}-3 \zeta^{2}\right)(4-\xi) \sin \left(4 \theta_{p q}\right)\right\} \sin \left(l \theta_{r p}\right)\right] .
\end{aligned}
$$




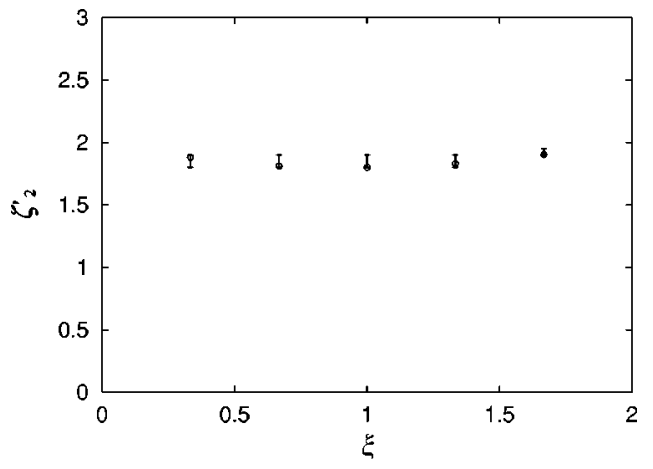

FIG. 2. The scaling exponents $\zeta_{2}^{\prime}$ of the homogeneous solutions with angular wave number $l=2$ of the simplified two-point closure equation. $\xi$ is the scaling exponent of the advecting field. $\lambda_{\xi, 2}^{\prime}(\zeta)$ changes its sign within the error bars and the circle symbols denote zero's obtained by a linear interpolation.

$\lambda_{\xi, l}(\zeta)$ in Eq. (33) is that of Eq. (23) with $\alpha=\gamma=1$. The integral in Eq. (33) converges when $\xi>0$ and $-|l-2|<\xi$ $<2-\xi+l$. Figure 2 shows the solution for $0<\xi<2$ and $l$ $=2$. The zero $\zeta_{2}^{\prime}$ of Eq. (32) is seen to be slightly smaller than 2 in the range $0<\xi<2$.

\section{CONCLUSIONS}

In this paper we proposed a model equation of a randomly advected solenoidal field that can be formally derived as a linearization of the Navier-Stokes equation [the model with parameters $(\alpha, \beta)=(1,1)$ in Eq. (4)]. The equation contains the pressurelike term which represents a spatially nonlocal dynamics. Unlike Kraichnan's model, the resulting closure equation of the second-order moments of the advected field contains not only derivatives but also integrals with respect to space variables.

In general, the scaling exponents of the homogeneous solutions of the closure equation are not determined from a dimensional analysis and take nontrivial values. An analysis is made of the scalings of the homogeneous solutions for both the isotropic part and the anisotropic part in $2 \mathrm{D}$ and it is shown that such nontrivial scaling exponents do exist. There is an isotropic homogeneous solution with the scaling exponent $\zeta_{0}=-\xi$. For the angular wave number $l=2$, two real exponents $\zeta_{2}^{(1)}$ and $\zeta_{2}^{(2)}$ which take nontrivial values in the range $(0,2)$ exist for $\xi_{2}^{c}<\xi<2$ where $\xi_{2}^{c} \approx 1.3$. A preliminary analysis for $l=4$ shows that there are two scaling exponents $2<\zeta_{4}^{(1)}<\zeta_{4}^{(2)}<4$ of the homogeneous solutions which are in agreement with "the law of isotropization.'

From the comparison of the models with the parameters $(\alpha, \beta)=(0,0) \quad$ (passive scalar), $(\alpha, \beta)=(-1,0) \quad$ (passive magnetic field), $(\alpha, \beta)=(0,1)$ (passive solenoidal vector without stretching), and $(\alpha, \beta)=(1,1)$, it is seen that the scaling exponent $\zeta_{0}$ of the isotropic part is not affected by the pressurelike term but depends alone on whether the stretching term is present. However, the scaling exponents $\zeta_{l}$ of anisotropic $(l \neq 0)$ homogeneous solutions are affected by the pressurelike term and it is suggested from the analysis for $l=2$ that the pressurelike term lowers the exponents $\zeta_{l}$ of the anisotropic part, i.e., the decay of anisotropy with the scale is slower under the existence of the pressurelike term.

The anisotropy of the second moment is also studied for a simplified two-point closure equation. The scaling exponent $\zeta_{2}^{\prime}$ of the anisotropy of $l=2$ is slightly smaller than 2 for 0 $<\xi<2$ and is smaller than that of Kraichnan's passive scalar model. Finally we note that the statistically stationarity of the model with parameter $(\alpha, \beta)=(1,1)$ is not yet solved and is left as a future problem.

\section{ACKNOWLEDGMENTS}

The authors are grateful to M. Nelkin L. Biferale, and K. Beronov for stimulating and useful discussions. This research was supported in part by the National Science Foundation under Grant No. PHY94-07194, during Y. K.'s participation in the program "Physics of Hydrodynamic Turbulence" at the Institute of Theoretical Physics (ITP), University California at Santa Barbara. This work was supported by the "Research for the Future" Program of the Japan Society for the Promotion of Science under Project No. JSPS-RFTF97P01101.
[1] M. Nelkin and T. Nakano, in Proceedings of IUTAM Symposium on Turbulence and Chaotic Phenomena in Fluids, Kyoto (Elsevier, Amsterdam, 1984), pp. 319-323.

[2] I. Arad et al., Phys. Rev. Lett. 81, 5330 (1998).

[3] I. Arad, V. L'vov, and I. Procaccia, Phys. Rev. E 59, 6853 (1999).

[4] R. H. Kraichnan, Phys. Fluids 11, 945 (1968).

[5] R. H. Kraichnan, Phys. Rev. Lett. 72, 1016 (1994).

[6] V. S. L'vov, I. Procaccia, and A. L. Fairhall, Phys. Rev. E 50, 4684 (1994).

[7] M. Chertkov, G. Falkovich, I. Kolokolov, and V. Lebedev, Phys. Rev. E 52, 4924 (1995).

[8] K. Gawȩdzki and A. Kupiainen, Phys. Rev. Lett. 75, 3634 (1995).
[9] A. Fairhall, O. Gat, V. L'vov, and I. Procaccia, Phys. Rev. E 53, 3518 (1996).

[10] U. Frisch, A. Mazzino, and M. Vergassola, Phys. Rev. Lett. 80, 5532 (1998)

[11] O. Gat, I. Procaccia, and R. Zeitak, Phys. Rev. Lett. 80, 5536 (1998).

[12] R. H. Kraichnan, Phys. Fluids 7, 575 (1965).

[13] Y. Kaneda, J. Fluid Mech. 107, 131 (1981).

[14] I. Arad, V. L'vov, E. Podivilov, and I. Procaccia, Phys. Rev. E (to be published).

[15] M. Vergassola, Phys. Rev. E 53, R3021 (1996).

[16] I. Arad, L. Biferale, and I. Procaccia, Phys. Rev. E 61, 2654 (2000). 